\title{
EL TAMAÑO MUESTRAL PARA LA TESIS.¿CUÁNTAS PERSONAS DEBO ENCUESTAR?
}

\section{THE SAMPLE SIZE IN THESIS. HOW MANY PEOPLE SHOULD I SURVEY?}

\author{
Villavicencio-Caparó Ebingen. ${ }^{1 *}$ Alvear-Córdova María Cristina. ${ }^{2}$ Cuenca-León Katherine. ${ }^{2}$ Calderón-Curipoma \\ Mireya. ${ }^{2}$ Zhunio-Ordoñez Karla. ${ }^{3}$ Webster-Carrión Frank. ${ }^{4}$ \\ ${ }^{1}$ Coordinador de Investigación de la Carrera de Odontología de la Universidad Católica de Cuenca.Ecuador \\ 2 Docente de la Carrera de Odontología de la Universidad Católica de Cuenca.Ecuador \\ 3 Odontóloga pasante de investigación en Odontología de la Universidad Católica de Cuenca.Ecuador. \\ ${ }^{4}$ Pasante de investigación en Odontología de la Universidad Católica de Cuenca.Ecuador \\ *ebingen@ hotmail.com
}

\begin{abstract}
Resumen
El presente artículo es una recopilación de los apuntes de la materia de investigación o bioestadística y de conferencias dictadas en latinoamérica. Al realizar una investigación científica uno de los principales factores que se debe tomar en cuenta es plantearse la interrogante de ¿Cuántas unidades de estudio se necesitarán para la realización del proyecto?, esta pregunta viene ligada a una segunda interrogante ¿Cómo calcular dicho tamaño de muestra?. El presente artículo de contribución didáctica docente pretende ser una guía con el fin de que los investigadores puedan tener un texto de referencia rápida para superar esta fase de la planificación de un estudio.
\end{abstract}

Palabras clave: Tamaño de la muestra, métodos, metodología, probabilidad.

\begin{abstract}
The article is a compilation of the notes of the subject of investigation or biostatistics and of lectures dictated in Latin America. When conducting scientific research one of the main factors to be taken into account is to ask the question of how many units of study will be needed to carry out the project?, this question is linked to a second question, How to calculate this sample size?. This bibliographic article aims to be a guide so that researchers can have a quick reference text to overcome this phase of planning a study.
\end{abstract}

Key words: Sample size, methods, methodology, probability.

\section{INTRODUCCIÓN}

Cuando planificamos realizar una investigación científica, en el paradigma cuantitativo, es imprescindible hacernos esta pregunta, ¿Cuántas unidades de estudio debo analizar?, muchas veces se cree que con 100 personas ya es suficiente, otras veces se plantea estudiar a todos los pacientes que acuden en dos meses a un centro de salud, otras veces se plantea la mitad de los alumnos de un salón como grupo experimental y la otra mitad como grupo control, todos estos criterios ayudan, pero no son los más adecuados, lo primero que debemos saber es cuántos grupos de pacientes tenemos: si es un solo grupo utilizaremos la fórmula para estimación de proporciones (diseño de estudio descriptivo) y si tenemos dos grupos de estudio utilizaremos la fórmula para comparación de proporciones( diseños de estudios de casocontrol, de cohortes o experimentales). Otra idea clave es que el número de unidades de estudio, está en íntima relación con el diseño del estudio (no confundir con diseño del proyecto) es decir con la estrategia operativa que vamos a emplear para responder a la pregunta de investigación. ${ }^{1}$ Entiéndase que los diseños de estudios también se pueden agrupar de acuerdo a la intervención del investigador en: diseños descriptivos, analíticos o experimentales y estos a su vez están en relación con la opción que uno toma para el manejo de las unidades de estudio, teniendo: ${ }^{2}$

- Opción población (cuando la totalidad de las unidades de estudio no supera las 499 unidades, se estudia a TODAS).

- Opción muestra (cuando la unidades son 500 o más, es 
eficiente la estrategia de muestreo).

- Opción grupos (cuando el estudio tiene más de un grupo de estudio y el objetivo es comparar la variable en ambos grupos).

- Opción estudio de caso. (Un solo caso o una serie de casos).

El diseño descriptivo, se adecua muy bien a la opción estudio poblacional y a la opción muestra. Los diseños analíticos y experimentales, se adecuan de mejor manera a la opción de grupos. ${ }^{3}$ Motivo por el cual en el presente artículo se hace una revisión de este tópico con la finalidad de aportar a quienes están realizando su proyecto de investigación.

\section{DESARROLLO}

En esta sección del artículo se desarrollarán las opciones muestra y grupos, con ejemplos didácticos para poder trasponer estas fórmulas a la investigación del lector:

\subsection{OPCIÓN MUESTRA DE UN GRUPO CON POBLA- CIÓN CONOCIDA}

En los estudios descriptivos donde se pretende hacer una investigación que incluya una muestra (un solo grupo de estudio, representativo de la población), el tamaño de muestra debe estar calculado en base a la probabilidad de que se dé el fenómeno estudiado, en la población que estamos investigando. ${ }^{4}$

Si el estudio no tiene antecedentes que nos hablen de la probabilidad de que ocurra este fenómeno, entonces asumimos que la probabilidad de que suceda este fenómeno es del $50 \%$, lo que en términos estadísticos se escribe como $\mathrm{P}=0,5$; de igual forma, tenemos que saber, cuál es el margen de error que nos permitiremos, convencionalmente se usa $5 \%$ que se escribe como $\mathrm{E}=0,05$; sabiendo que la confiabilidad tiene una constante de $\mathrm{Z}=1,96$; para el $95 \%$ de confianza, finalmente solo nos queda averiguar el tamaño de la población $(\mathrm{N})$ y aplicamos la siguiente fórmula para estimar el tamaño de la muestra:

El lector podrá aplicar esta fórmula a su caso particular y saber inmediatamente cuál es el tamaño de su muestra y por consiguiente a cuántas personas incluir en el estudio. Este tipo de muestreo también se puede utilizar en los estudios relacionales, con un solo grupo de unidades de estudio y donde se miden dos variables en la misma persona.

\subsection{OPCIÓN MUESTRA DE UN GRUPO CON POBLA- CIÓN DESCONOCIDA}

Un segundo ejemplo sería en el caso en el que no se cuente con el tamaño de la población, para este escenario debemos utilizar la siguiente fórmula:

Donde:

Z: 1,96 constante para el $95 \%$ de confiabilidad

$$
n=\frac{Z^{2} * P(1-P) * N}{Z^{2} * P *(1-P)+E^{2} *(N-1)}
$$

Fórmula lista para reemplazar:

$$
n=\frac{1,96^{2} * 0,5(1-0,5) * N}{1,96^{2} * 0,5 *(1-0,5)+(0,05)^{2} *(N-1)}
$$

Fig. 1.

$$
n=\frac{Z^{2} * P *(1-P)}{E^{2}}
$$

\section{Fórmula lista para reemplazar:}

$$
n=\frac{1,96^{2} * 0,5(1-0,5)}{(0,05)^{2}}=384
$$

Fig. 2.

P: 0,5 probabilidad que se dé el evento esperado 1-P: Es $1-0,5=0,5$

\subsection{OPCIÓN GRUPOS PARA VARIABLE CUALITATIVA .}

En los estudios analíticos donde se pretende hacer una investigación que incluya dos muestras (dos grupos de estudio, caso-control o grupo experimental- grupo control), el tamaño de muestra debe estar calculado en base a la diferencia entre ambos grupos, motivo por el cual, acá no funciona la fórmula anterior y aplicaremos una nueva más sencilla. Los insumos necesarios son: (P1) que es la probabilidad de que el fenómeno se dé como espera el investigador en el primer grupo (grupo control) y la probabilidad (P2) que significa la probabilidad que se dé el fenómeno en el segundo grupo (grupo experimental), tal como espera el investigador. ${ }^{6}$ Generalmente P1 se obtiene de los antecedentes de investigación, si no hay antecedentes se asume P1=0,5 y P2 se obtiene DE LA HIPÓTESIS DEL 
INVESTIGADOR, en este caso asumimos P2=0,95; motivo por el cual P2, queda al criterio del investigador, luego tenemos dos constantes una que es $\mathrm{Za}=1,96$ referida al error tipo I y otra que es $\mathrm{Zb}=1,64$; referida al error tipo II veamos cómo queda la fórmula.

$$
n=\left(\frac{Z a \sqrt{2 P(1-P)}+Z b \sqrt{P_{2}\left(1-P_{2}\right)+P_{1}\left(1-P_{1}\right)}}{P_{1}-P_{2}}\right)^{2}
$$

Fig. 3 .

\section{Donde:} tipo I)

Za: 1,96 constante para el $95 \%$ de confiabilidad (error Zb: 0,84 constante para el $80 \%$ potencia (error tipo II)

P1: 0,5 probabilidad que se dé el evento esperado en el grupo control o placebo.

P2: 0,95 probabilidad que se dé el evento esperado en el grupo experimental o de expuestos.

Fórmula con datos reemplazados:

$$
n=\left(\frac{1.96 \sqrt{2(0,725)(1-0,725)}+0,84 \sqrt{(0,95)(1-0,95)+(0,5)(1-0,5)}}{0,5-0,95}\right)^{2}
$$

Fig. 4.

El lector podrá aplicar esta fórmula a su caso particular y saber inmediatamente cuánto es el tamaño de su muestra y por consiguiente a cuántas personas incluir en el estudio. El resultado de esta fórmula es para cada grupo, es decir en el ejemplo presentado el resultado es 14,22 que se redondea a 14 unidades de estudio, motivo por el cual; se tendrían que tomar 14 pacientes en el grupo experimental y 14 pacientes en el grupo control. ${ }^{7}$

\subsection{OPCIÓN GRUPOS PARA VARIABLE CUANTITATI- $V A$.}

En el caso de las investigaciones que miden una variable numérica en dos grupos, con la finalidad de comparar (ej. filtración marginal, índice CPOD, profundidad al sondaje) se debe utilizar la siguiente fórmula.

Donde:

Za: 1,96 constante para el $95 \%$ de confiabilidad (error tipo I) Zb: 0,84 constante para el $80 \%$ potencia (error tipo II)

$S^{2}$ : Varianza de la variable en el grupo control, reportado en los estudios de referencia, para el ejemplo hemos tomado la desviación estándar del CPOD (2.2)de un estudio previo. ${ }^{8}$

$$
n=\frac{2(Z \alpha+Z \beta)^{2} * \delta^{2}}{e^{2}}
$$

Fig. 5.

$e^{2}$ : Diferencia entre el promedio del grupo 1 con el promedio del grupo 2, obtenido en el piloto o por antecedentes, para este ejemplo hemos sacado la diferencia de CPOD del grupo 1 (4.32) con el CPOD del grupo 2 (5.22) de dos estudios previos. $^{8}$

Reemplazando la fórmula tenemos:

$$
n=\frac{2 *(1,96+0,84)^{2} * 2,2^{2}}{0,9^{2}}=38,32
$$

Fig. 6.

En este ejemplo se ha utilizado una desviación estándar de 2,2 que al elevarla al cuadrado se convierte en varianza y una diferencia de promedios de CPOD de dos ciudades que equivale a 0.9 ; el resultado nos indica 38,2 ; que significa que debemos considerar 38 pacientes por cada grupo para un posterior estudio comparativo de CPOD entre dos grupos de pacientes.

\section{CONCLUSIONES}

El tamaño de la muestra está relacionado, con el tipo de estudio, en el caso de estudios descriptivos y relacionales, el objetivo es tener un tamaño de muestra que sea representativo de la población y la potencia del estudio está relacionada de manera proporcional con el tamaño de la muestra. Si este tamaño es adecuado, las conclusiones que se logren del estudio de esta muestra, son extrapolables a la población original.

El objetivo de hallar el tamaño de muestra en los estudios analíticos, está relacionado a poder hacer la comparación entre dos grupos, motivo por el cual debemos tener en antecedentes investigativos, los datos del grupo control y ahí radica la importancia de los estudios descriptivos, pues al terminar un estudio descriptivo logramos como dato final el valor de P1 que es el insumo más importante en la fórmula del tamaño de muestra del estudio experimental, de caso control o de cohortes(que utilizan la fórmula de comparación de proporciones). Finalmente se debe mencionar que una muestra adecuada, le da la potencia necesaria al estudio y 
que las conclusiones que logremos de ese estudio, serán científicamente válidas.

Conflicto de intereses y financiamiento Los autores declaran no tener conflicto de , haber cumplido con los requisitos de autoría y haber autofinanciado este artículo.

\section{Referencias}

1 Wayne W. Daniel; Bioestadística bases para el análisis de las ciencias de la salud, Edit. Limusa México 1985.

2 Villavicencio-Caparó E. Cuenca-León K. Vélez-León E. Sayago-Heredia J. Cabrera-Duffaut A. Pasos para la planificación de una investigación clínica. Odontología Activa, v. 1, n. 1, p. 75-78, jun. 2016. ISSN 2588-0624.

3 Francisco J. Barón L. Apuntes de bioestadística: tercer ciclo en ciencias de la salud y medicina: Universidad de Málaga España 2005.

4 Hernández Sampieri R. Metodología de la investigación, Edit. Mc Graw- Hill México 1991.

5 Murray R. Spiegel; Estadística,Edit. Mc Graw- Hill Colombia 1970 .

6 Dawson B. Bioestadística médica: 3era edición, Edit. Manual Moderno México 2002.

7 Geoffrey R. Norman, David L. Streiner. Bioestadística: Edit. Harcourt Brace España 1998.

8 Reinoso Vintimilla, N. Villavicencio-Caparó, E. Caries dental en escolares de 12 años de la parroquía Sayausí (Cuenca) Ecuador. Odontología Activa, V. 1, N. 1, p. 34-39, jun. 2016. ISSN 2588-0624.

Recibido: 1 de Enero de 2017

Aceptado: 30 de Enero de 2017 